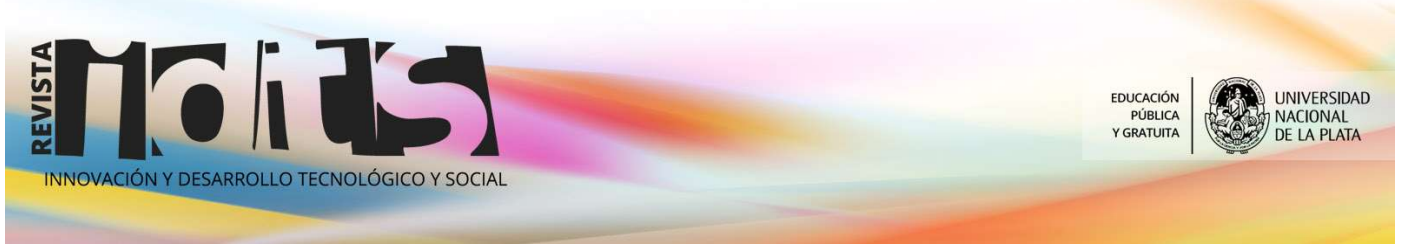

Innovación y Desarrollo Tecnológico y Social (2019) 1 (2): 36-43

\title{
Uso de magnetometría terrestre en la exploración de pelitas ferruginosas en canteras del Distritominero Barker, provincia de Buenos Aires
}

\author{
Tessone, Mario ${ }^{1,3}$; Nigro, Joaquín ${ }^{1}$; Perdomo, Santiago ${ }^{1,2}$; Etcheverry, Ricardo ${ }^{1,2}$; \\ Fernández, Raúl ${ }^{1,3}$;Tessone Nicolás ${ }^{2}$; Caballé, Marcelo ${ }^{1}$ \\ ${ }^{1} U N L P ;{ }^{2} C O N I C E T ;{ }^{3} C I C B A$ \\ ${ }^{3}$ mtessone@inremi.unlp.edu.ar
}

Resumen: En la Sierra de La Juanita y la Cuchilla de las Águilas, cercanas a la localidad de Barker, se extraen rocas ferruginosas destinadas a la industria del cemento. La falta de continuidad lateral de los bancos constituye un problema para los productores, tanto en la planificación de la explotación como de la recuperación ambiental. Con el fin de contribuir a la solución o mitigar esta problemática se aplicaron técnicas geofísicas y a partir de ello se realizaron reinterpretaciones geológicas de las áreas. Sobre la base de la información de superficie se planteó la utilización de un método geofísico rápido y de bajo costo, consistente en mediciones de susceptibilidad magnética en las rocas de canteras seleccionadas. Comprobada la respuesta positiva de los niveles ferriferos por sus valores elevados de susceptibilidad, se programó una exploración magnetométricaterreste en el área de influencia de las canteras, con el fin de evaluar su eficacia para detectar en el subsuelo capas con altos tenores de Hierro y ajustar el tratamiento de los datos para la obtención de resultados óptimos.

Palabras Claves: magnetrometría; exploración; arcillas; recuperación ambiental; Barker

Recibido 04/06/2019 Aceptado 22/11/2019

DOI $10.24215 / 26838559 \mathrm{e} 009$

\section{Use of terrestrial magnetometry in the exploration of ferruginous pelites in quarries of the Barker mining district, province of Buenos Aires.}

\begin{abstract}
Ferruginous pelites mainly destined for the cement industry are extracted in the Sierra de La Juanita and Cuchilla de lasÁguilas, near Barker town. The lack of lateral continuity of the pelitic banks constitutes a problem for the producers, both in the planning of the exploitation and of the related environmental recovery. In order to contribute to the solution or mitigate this problem, geophysical techniques were applied, which allowed geological reinterpretations to be done. Based on surface information, the use of a fast and low-cost geophysical method was proposed. The latter consisted of magnetic susceptibility measurements in selected quarry rocks. Once the positive response of the ferriferous levels was verified due to their high susceptibility values, a terrestrial magnetometric exploration in the quarries influence areas was programmed, in order to evaluate its effectiveness to detect underground layers with high iron levels and adjust data processing for optimal results.
\end{abstract}

Keywords: magnetometry, exploration, clay, environmental recovery, Barker 


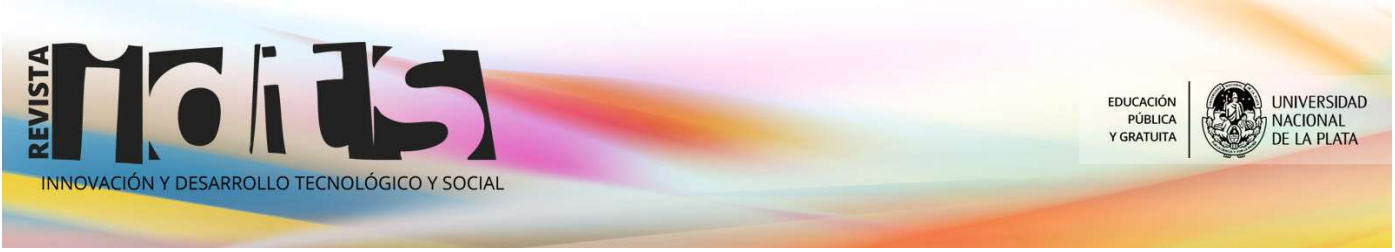

Innovación y Desarrollo Tecnológico y Social (2019) 1 (2): 36-43

\section{Novedad u originalidad local en el conocimiento}

El Distrito Barker tiene gran importancia en la minería bonaerense, especialmente por la explotación de calizas y arcillas, mayormente utilizadas en la industria del cemento y, las segundas, también en la elaboración de productos cerámicos. Como ocurre en general con la producción de rocas de aplicación y minerales industriales, la utilización de métodos geofísicos para detectar recursos no expuestos en superficie es casi inexistente. No es costumbre de los pequeños productores mineros utilizar métodos geofísicos para la prospección y/o exploración. Esto se debe, al menos en parte, a la falta de información adecuada sobre eficacia $v s$ costos. La medición de la susceptibilidad magnética se realiza a través de un método sencillo, novedoso a nivel local, que ha permitido detectar concentraciones de rocas ferríferas en profundidad. Su utilización no implica grandes inversiones sino, al contrario, permite reducir los gastos de laboreo superficial y también los costos ambientales.

\section{Grado de relevancia}

Dada la importancia económica de estas concentraciones de Fe en rocas pelíticas y la problemática ambiental asociada a la extracción, la relevancia del trabajo radica en haberse comprobado la eficacia del método para mejorar sensiblemente el manejo del recurso, a partir del conocimiento de la ubicación de los mantos en profundidad. La información obtenida resulta útil para realizar una explotación racional desde el punto de vista productivo, con disminución del impacto ambiental. Es decir que su uso produciría ventajas económicas a las empresas productoras al disminuir los laboreos en superficie y, al mismo tiempo, traería aparejada una reducción del impacto en el ambiente, especialmente el de carácter geomorfológico y paisajístico.

\section{Grado de pertinencia}

Si bien los trabajos realizados fueron por ahora de carácter preliminar, los resultados obtenidos permitieron comprobar la eficacia del método y la conveniencia de su aplicación en otros casos similares. La disminución de los movimientos de tierra y de labores superficiales redundarán, como se ha dicho, en beneficios para las empresas, pero además acompañarían las políticas de cuidado ambiental que lleva adelante el Municipio de Benito Juárez.

Los trabajos fueron financiados mediante subsidios de I+D de la UNLP y PDTS CIN-CONICET. Las empresas propietarias de las minas han adoptado los resultados para la definición y apertura de sus próximos frentes de explotación, encontrándose también interesadas en aplicar esta metodología para la planificación de sus próximos trabajos de prospección y exploración. La metodología puede ser extensiva a las canteras de la mayoría de los productores de pelitas ferruginosas de la región, en general con alta demanda para la producción de cemento.

\section{Desarrollo del producto}


Las canteras analizadas en la zona de Barker son La Ramona, Martín Fierro y La Bermeja, emplazadas en la Sierra de La Juanita, y las denominadas La Elisa, La Carreta y San Jacinto ubicadas en la Cuchilla de las Águilas (Fig. 1). Se localizan, respectivamente, a unos $10 \mathrm{~km}$ al este y al oeste de la localidad de Barker. Se trata de explotaciones de pelitas ferruginosas, correspondientes a la Formación Las Águilas (Zalba, 1978;Poiré y Spalletti, 2005). Las áreas estudiadas fueron seleccionadas por la representatividad geológica expuesta en los distintos frentes de explotación.

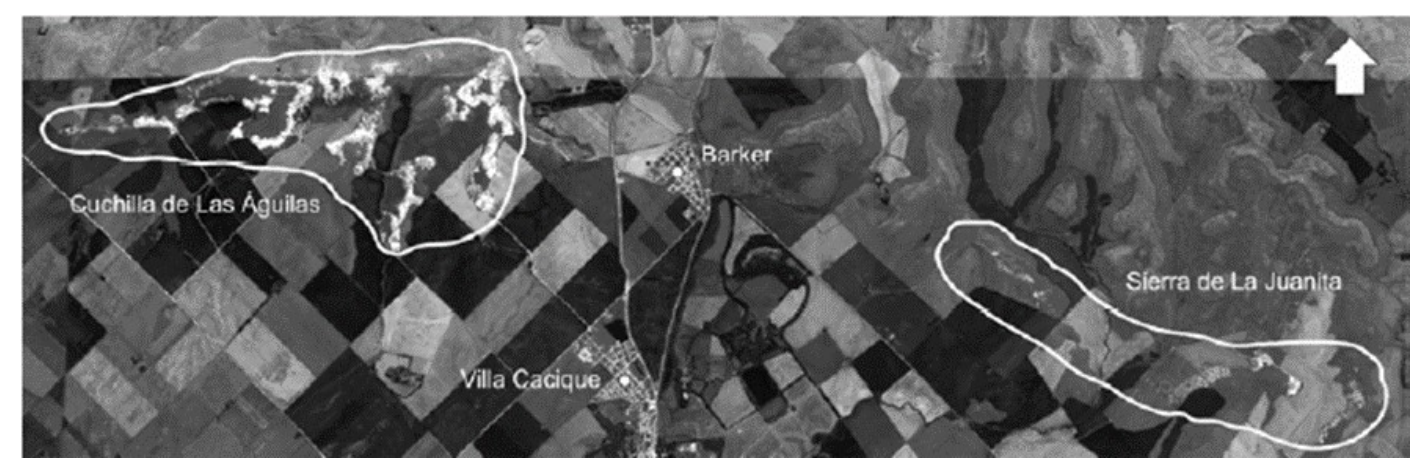

Figura 1: Ubicación del área de estudio

Figure 1: Study area

La falta de continuidad lateral de los niveles ferríferos y el desconocimiento geológico del subsuelo, constituyen los mayores problemas en la explotación, hecho que hasta el momento ha llevado a los productores a avanzar en los trabajos “a ciegas" , sin una guía clara de ubicación de los sectores de arcilla ferruginosa en profundidad.

Actualmente las empresas mineras locales explotan bancos o niveles ricos en $\mathrm{Fe}$ que ofrecen espesores significativos ( $>$ a $2 \mathrm{~m}$ ). De acuerdo al sistema elemental que utilizan, estos espesores les posibilitan una adecuada apertura de los frentes de cantera. Para remover el estéril superior y detectar los sectores de interés en el subsuelo generan huecos extremadamente amplios, ya que los cambios laterales dificultan la localización previa de los sectores más aptos (de mayor espesor y concentración de Fe) donde centralizar la exploración y posterior explotación. Esta búsqueda, muchas veces azarosa, además produce un desmedido impacto paisajístico y geomorfológico en las sierras.

La interpretación de los perfiles de susceptibilidad magnética realizados permitió determinar con aceptable precisión la situación espacial, profundidad y espesor de los niveles ferruginosos.Por otra parte, muestreos de detalle y análisis químicos orientativos determinaron contenidos de óxidos de hierro de hasta el 70\% en las rocas (Etcheverry et al., 2010).

Los niveles de interés se encuentran a una profundidad variable entre 15 y 20 metros. La discontinuidad lateral de los bancos hace que se pase de sitios con espesores comerciales a no comerciales en corta distancia.

Actualmente el método de exploración contempla, a lo sumo, un programa de perforaciones y la apertura de excavaciones con maquinaria pesada, lo cual resulta un proceso lento y costoso tanto económica como ambientalmente. La disminución del tamaño y de la cantidad de labores mineras 
para la búsqueda redundaría en una reducción de los costos y una minimización del impacto ambiental minero.

La primera etapa del trabajo consistió en la determinación de la susceptibilidad magnética de las distintas litologías presentes en diferentes sectores de las canteras, llevada a cabo mediante la utilización de un Susceptibilímetro MS2D de Bartington Instruments. Se utilizó un método diferencial, con un magnetómetro en una estación fija tomando mediciones con intervalos de 3 segundos y otro móvil con la misma frecuencia de lectura, ambos sincronizados temporalmente al inicio de las tareas diarias. El equipo base es de marca Scintrex, modelo EnviMag (Fig. 2) y el móvil es marca GemSystem modelo GSM-19.Analizados los datos, se constató que los niveles ferruginosos presentan un alto contraste de susceptibilidad magnética respecto a las otras litologías asociadas. Comprobada la pertinencia del método se realizó el estudio de magnetometría terrestre mediante itinerarios regulares (33.500 metros), con líneas separadas por 50 metros con un acimut de $160^{\circ}$. Los recorridos cubrieron las zonas de interés, rodeando e interviniendo las canteras de las cuales se extraen las pelitas ferruginosas.

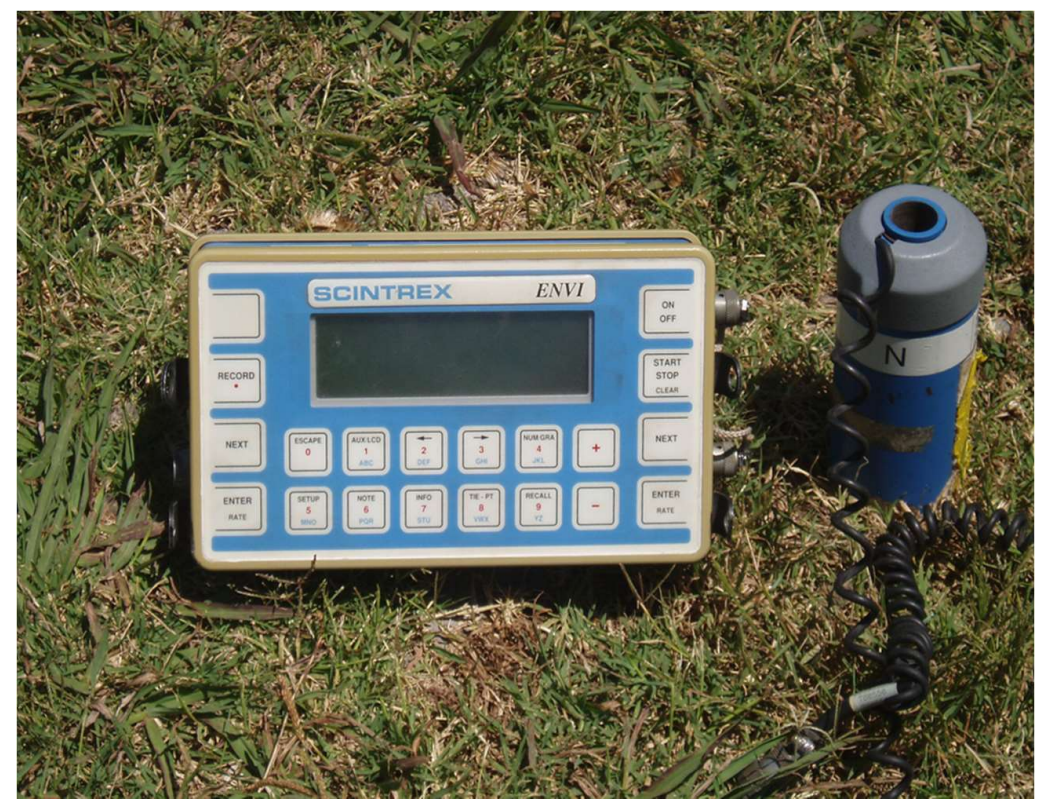

Figura 2: Magnetómetro protónico ENVI Mag

Figure 2: Protonmagnetometer ENVI Mag

Como se observa en la Tabla 1, las mediciones de susceptibilidad magnética marcan un contraste significativo entre las pelitas ferruginosas, las denominadas pelitas blancas y varicolores y las areniscas cuarcíticas.

El contraste observado permitió plantear el estudio magnetométrico terrestre principalmente para aquellos sectores que aún no fueron explotados, para lo cual se partió de los frentes de canteras donde se observan pelitas ferruginosas (Fig. 3). 
INNOVACIÓN Y DESARROLLO TECNOLÓGICO Y SOCIAL

Innovación y Desarrollo Tecnológico y Social (2019) 1 (2): 36-43

Tabla 1. Mediciones de susceptibilidad magnética con promedios

Table 1. Measurements of magnetic susceptibility to averages

\begin{tabular}{|c|c|c|c|}
\hline Litología & $\begin{array}{c}\text { Cantidad de } \\
\text { mediciones }\end{array}$ & $\begin{array}{c}\text { Rango: min }- \text { max } \\
(\operatorname{cgs~10-6})\end{array}$ & $\begin{array}{c}\text { Promedio } \\
(\operatorname{cgs~10})\end{array}$ \\
\hline Areniscas cuarcíticas & 13 & $1,0-1,5$ & 1,317 \\
\hline $\begin{array}{c}\text { Pelitas varicoloreso } \\
\text { psamo-pelitas }\end{array}$ & 16 & $0,80-0,96$ & 0,858 \\
\hline $\begin{array}{c}\text { Pelitas ferruginosas } \\
\text { laminadas }\end{array}$ & 12 & $10,0-11,0$ & 10,208 \\
\hline $\begin{array}{c}\text { Pelitas ferruginosas } \\
\text { masivas }\end{array}$ & 16 & $16,0-18,0$ & 17,209 \\
\hline
\end{tabular}

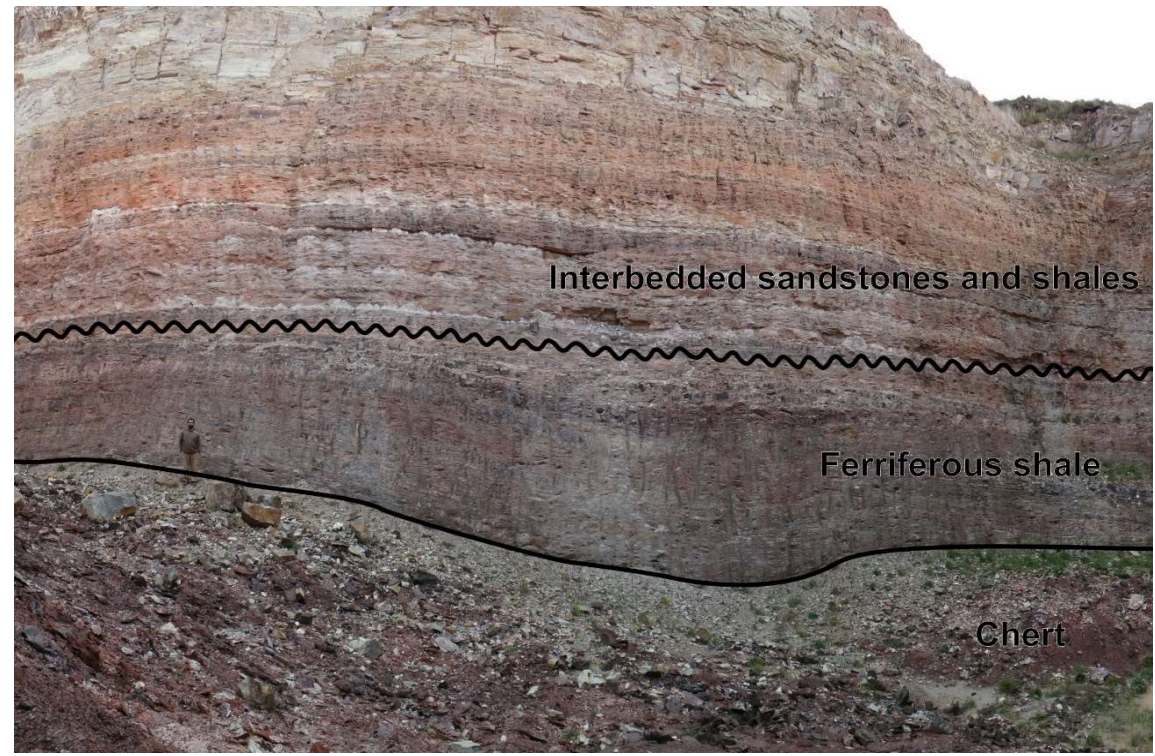

Figura 3. Frente en la cantera La Ramona

Figure 3. Front in La Ramona quarry

La interpretación de los resultados aportados por la magnetometría manifiesta, en general, una coincidencia espacial entre las anomalías magnéticas y los niveles de pelitas ferruginosas observados en los frentes de las canteras en explotación. Del mismo modo, se deduce una parcial continuidad de estas anomalías en el sentido del buzamiento de los mismos, en dirección sudoeste, y según el ensayo de modelo realizado se interpreta un aumento en los espesores en igual dirección (Fig. 4). 


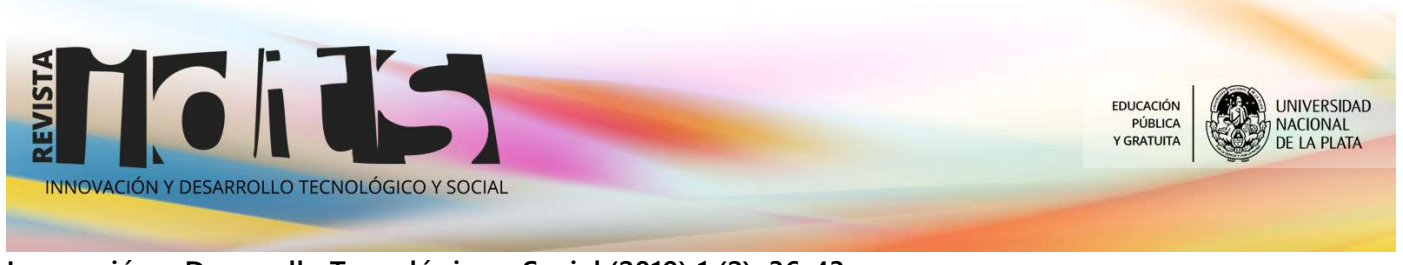

Innovación y Desarrollo Tecnológico y Social (2019) 1 (2): 36-43

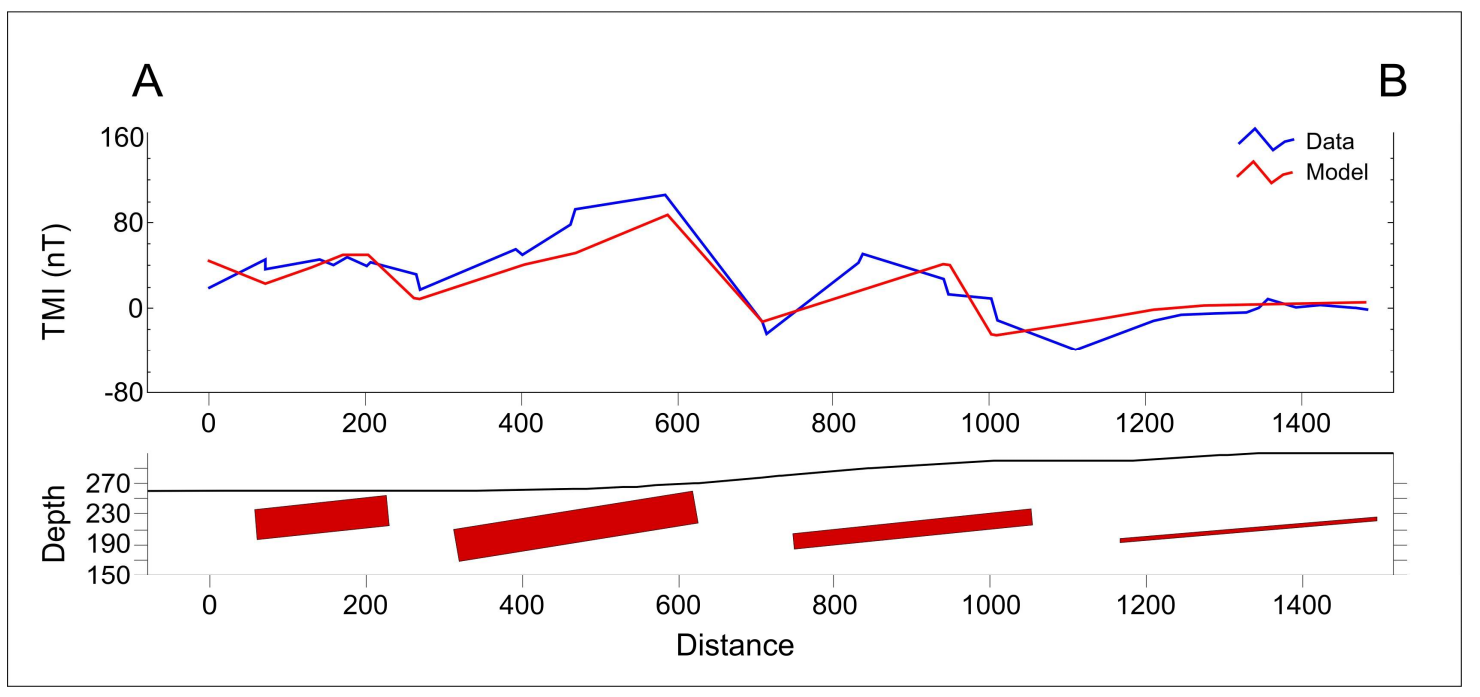

Figura 4. Modelado a partir de los datos de campo total

Figure 4. Modeling from the total field data

Sobre la base del marco geológico de la región de estudio y a partir de los datos del campo total tomados en el terreno y su modelización se puede interpretar la evolución del yacimiento, con una primera etapa de depositación uniforme de chert y pelitas ferruginosas suprayacentes y la posterior deformación tectónica que originó bajos y altos estructurales. Las discontinuidades entre las anomalías magnéticas observadas en la modelización de los datos, se podrían explicar a partir del desarrollo de una superficie de discordancia. Principalmente en los altos estructurales se produjeronfenómenos erosivos de los niveles más friables (pelitas con $\mathrm{Fe}$ ), quedando en los núcleos de los anticlinales las pelitas con Chert, como relictos de erosión, mientras que los núcleos de los sinclinales preservaron los mayores espesores de las pelitas ferruginosas, coincidentes con las anomalías magnéticas. Posteriormente la depositación de la secuencia psamo-pelítica y finalmente el basculamiento regional de la secuencia ya estructurada (Fig.5). 


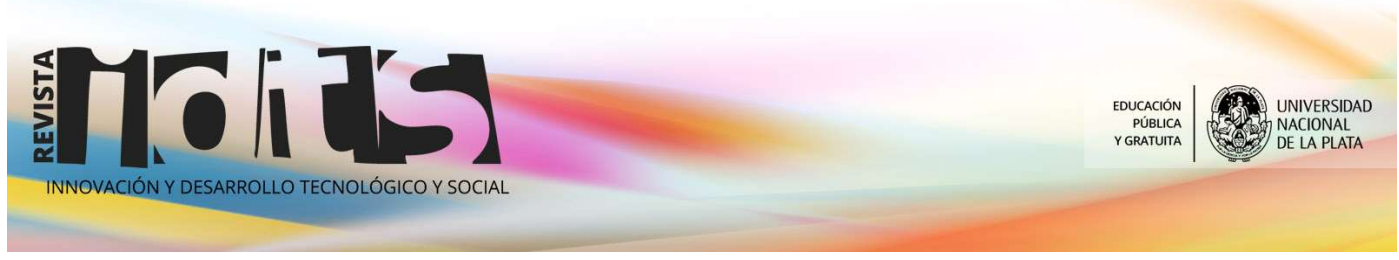

Innovación y Desarrollo Tecnológico y Social (2019) 1 (2): 36-43

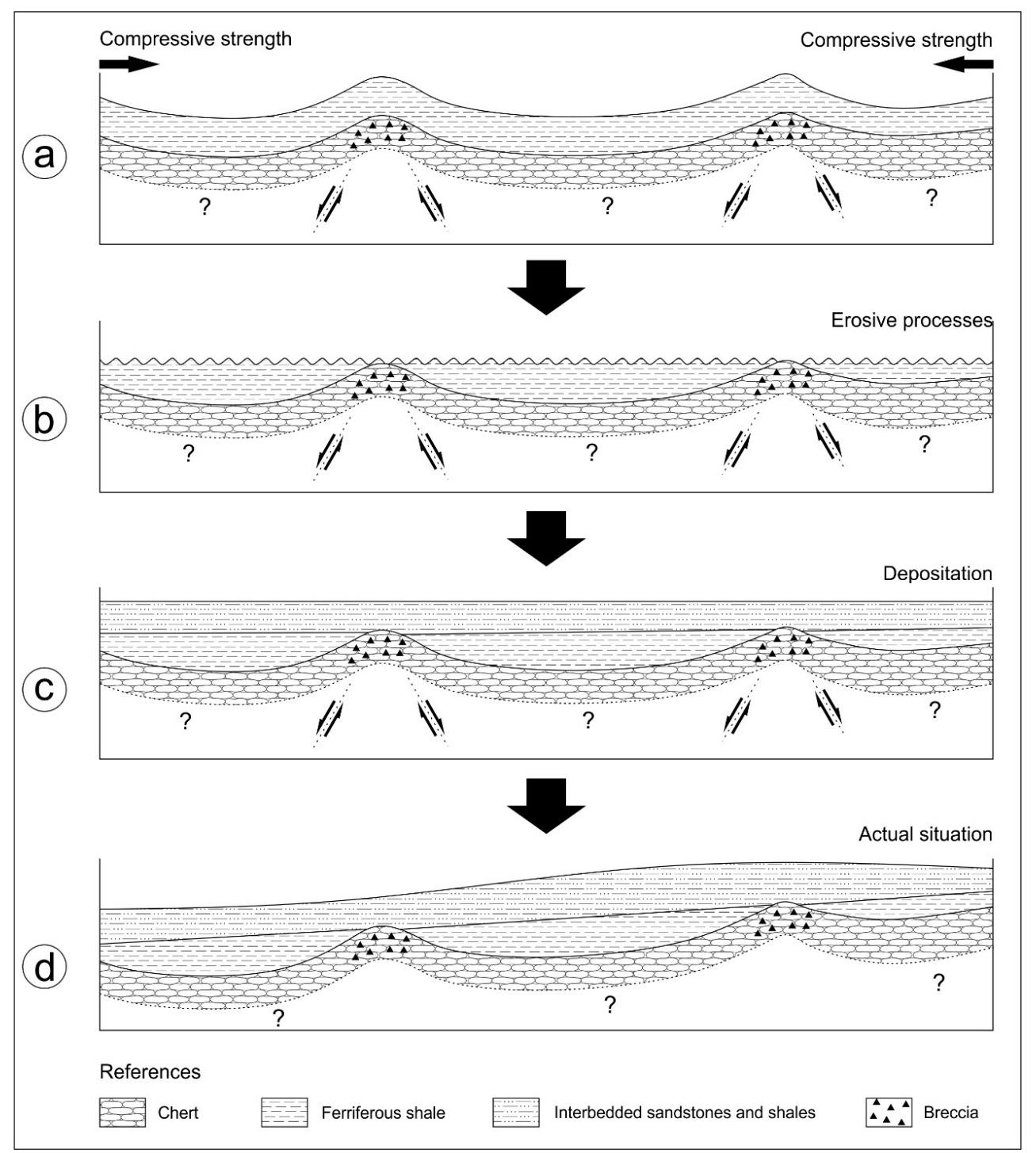

Figura 5. Esquema interpretativo de la evolución sedimentaria y estructural del área

Figure 5. Interpretative scheme of the sedimentary and structural evolution of the area

\section{Conclusiones}

La aplicación de métodos magnetométricos terrestres de detalle para la prospección-exploración de pelitas ferruginosas resulta apropiada para la región de Barker, ya que se manifiestan importantes contrastes de susceptibilidad magnética entre las pelitas ferruginosas y el resto de las rocas sedimentarias que componen la estratigrafía de la región. No se descarta que también sea aplicable en otras zonas geológicamente similares en el marco geológico de las Sierras Septentrionales de la provincia de Buenos Aires. 


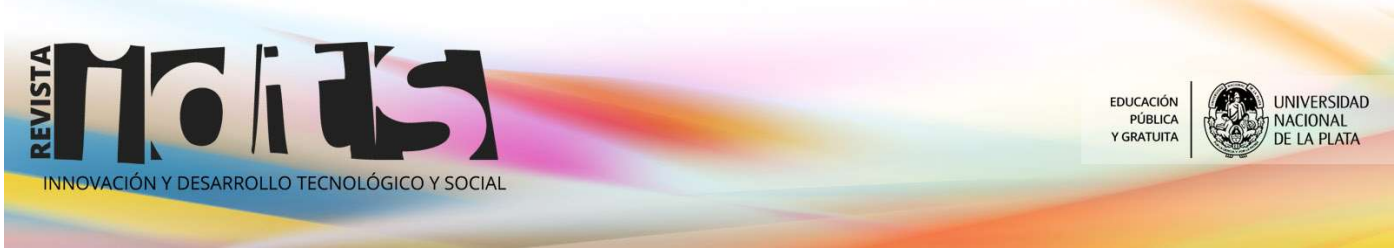

Innovación y Desarrollo Tecnológico y Social (2019) 1 (2): 36-43

Las discontinuidades laterales de la respuesta anómala magnética observadas en el modelo ensayado, se atribuyen a la falta de continuidad de los mantos de las pelitas ferruginosas debido a la deformación tectónica y la posterior erosión de los niveles más friables, que generó una discordancia entre ellas y las unidades sedimentarias sobrepuestas.

La modelización de las anomalías magnéticas de los mantos de pelitas ferruginosas permitiría realizar una mejor planificación de futuras explotaciones, disminuyendo las excavaciones en sectores estériles o con poca potencialidad minera y por lo tanto los costos de explotación. Consecuentemente, también se lograría mejorar la recuperación ambiental de los terrenos afectados por el laboreo minero.

\section{Financiamiento y colaboración}

El presente desarrollo fue realizado con fondos de los siguientes subsidios: UNLP 11N666, UNLP 11 N801, CIN-CONICET PDTS $n^{\circ}$ 409. Se contó con la colaboración de distintos productores mineros que facilitaron el ingreso a sus canteras y ejecutaron laboreos exploratorios para la aplicación del método propuesto.

\section{Bibliografía}

Etcheverry, R., Tessone, M. Caballé, M. y Fernández, R., 2010. Niveles de arcillas ferruginosas en las Sierras Septentrionales de Buenos Aires, Argentina. Su aprovechamiento industrial. En VII Jornadas Iberoamericanas de Materiales de Construcción. Organizado por Escuela Técnica Superior de Ingenieros de Minas de Madrid. Lima, Perú, agosto del 2010.

Poiré D. y Spalletti L., 2005. La cubierta sedimentaria precámbrica-paleozoica inferior del Sistema de Tandilia. En: R.E de Barrio, R.O Etcheverry, M.F Caballé y E. Llambías (Eds), Geología y recursos minerales de la provincia de Buenos Aires. Relatorio16 ${ }^{\circ}$ Congreso Geológico Argentino: 51-68.

Zalba P.E., 1978. Estudio geológico-mineralógico de los yacimientos de arcillas de la zona de Barker, partido de Juárez, provincia de Buenos Aires y su importancia económica. Tesis Doctoral 362, Facultad de Ciencias Naturales y Museo, Universidad Nacional de La Plata, 75pp. (Inédito). 